\title{
Relación entre niveles de carbamazepina en saliva y plasma: Estudio piloto
}

\author{
Sigrid Mennickent $\mathrm{C}^{\mathrm{la}}$, Mario Vega $\mathrm{H}^{2 \mathrm{~b}}$, \\ Carmen G loria Godoy $\mathbf{M}^{1 \mathrm{C}}$, M. D oris León $\mathbf{H}^{3}$. \\ Saliva and plasma levels of \\ carbamazepine have a poor \\ correlation: a pilot study
}

\begin{abstract}
Carbamazepine is one of the most commonly used anticonvulsants for the treatment of epilepsy and its plasma concentrations must be monitored periodically to obtain a useful and safe clinical effect. There is not a good relationship between the dose of the carbamazepine and their effects in humans, but the effects of this drug have been well correlated with its plasma levels. Aim: To measure the correlation between plasma and saliva levels of carbamazepine in children with epilepsy. Material and Methods: Saliva and plasma levels of carbamazepine were measured by using instrumental planar chromatography in 11 epileptic children aged 8 to 15 years treated with the drug for at least six months. Results: The mean saliva/plasma ratio was $0.18 \pm 0.05$ and the mean of carbamazepine concentration in saliva, expressed as a percentage of concentrations in plasma, was $17.97 \pm 5.40$. There was a poor linear correlation $(r=0.37)$ between the concentrations of carbamazepine in both fluids. Conclusions: In this group of epileptic children the correlation between saliva and plasma carbamazepine levels was weak (Rev Méd Chile 2007; 135: 335-40). (Key w ords: Carbamazepine; Epilepsy; Saliva)

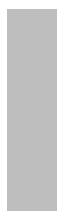

\footnotetext{
${ }^{1}$ Departamento de Farmacia, Facultad de Farmacia, Universidad de Concepción. ${ }^{2}$ Departamento de Bromatología, Nutrición y Dietética, Facultad de Farmacia, Universidad de Concepción, Chile. ${ }^{3}$ Policlínico de Neurología, Hospital Las Higueras, Talcahuano. aQuímico Farmacéutico, Magíster en Ciencias Farmacéuticas

${ }^{\mathrm{b}} \mathrm{Químico}$ Farmacéutico, Magíster en Nutrición Humana

'Químico Farmacéutico, Doctor en Farmacia.
}

L a epilepsia es un desorden crónico del sistema nervioso central caracterizado por ataques recurrentes y autolimitados. Periódicamente, las neuronas depolarizan en forma anormal, causan-

$\overline{\text { Correspondencia a: Sigrid Mennickent C. Departamento de }}$ Farmacia, Universidad de Concepción. Casilla 237, Concepción, Chile. Fono: 56-41-2204523; Fax: 56-41-2207086. E mail: smennick@udec.cl do alteraciones en la actividad motora, sensorial o en el comportamiento ${ }^{1,2}$.

La epilepsia afecta a 2,3 millones de personas tan sólo en Estados Unidos de Norteamérica, produciéndose en dicho país alrededor de 181.000 nuevos casos cada año ${ }^{1}$.

Sumado a las complicaciones de los ataques, los pacientes pueden experimentar efectos indeseables producidos por los medicamentos anti- 
convulsivantes (antiepilépticos). La mayoría de los tratamientos de la enfermedad persiguen lograr un equilibrio entre la prevención de los ataques y el intento de disminuir al mínimo el riesgo de efectos laterales de los fármacos ${ }^{1,2}$. Estos pueden producir falla en el tratamiento o pobre adherencia a éste. Además, pueden conducir a la hospitalización del paciente, con el consiguiente desmedro de su salud y costo económico ${ }^{1}$. Estos efectos indeseables pueden variar en gravedad, desde trastorno mínimo del sistema nervioso central hasta muerte por anemia aplástica 0 insuficiencia hepática ${ }^{2}$.

El control de los niveles plasmáticos de los fármacos facilita en gran medida el lograr una medicación anticonvulsivante óptima, sobre todo cuando se inicia el tratamiento, después de ajustes posológicos, en caso de fracaso, cuando se manifiestan efectos tóxicos, o cuando se instituye tratamiento con nuevos medicamentos ${ }^{2}$.

La carbamazepina es un medicamento primario para el tratamiento de las convulsiones parciales y tonicoclónicas ${ }^{2-4}$. Además, en la actualidad es, junto a la oxcarbazepina, el compuesto de elección en el tratamiento de la neuralgia del trigémino y glosofaríngea ${ }^{2,5}$, y se utiliza también en trastornos bipolares $2,5,6$. Su mayor uso en epilepsia es en niños ${ }^{5}$, y en ellos los principales efectos adversos de este fármaco son somnolencia, pérdida de coordinación y vértigo. Efectos más serios incluyen trastornos hematológicos, dermatológicos, hepáticos y pancreáticos ${ }^{2,4,5}$. Este medicamento presenta una baja solubilidad, además de un metabolismo inducido por otros fármacos y también por autoinducción. Esto genera una pobre relación entre dosis y efecto, lo cual, sumado al riesgo de efectos adversos, aconseja el monitoreo de sus concentraciones plasmáticas para obtener regímenes terapéuticos seguros y efectivos ${ }^{2,7}$.

Carbamazepina difunde bien al líquido cefalorraquídeo, fluidos duodenales, bilis, saliva y leche ${ }^{3,7,8}$. Sin embargo, el monitoreo de este fármaco se realiza en plasma y suero ${ }^{9-15}$. La determinación de niveles de fármacos en saliva presenta ventajas considerables, como son que la recolección de la muestra es fácil y no invasiva, logrando así una mayor adherencia de los pacientes a los controles de niveles de sus medicamentos. En niños, el muestrear en saliva no pone en riesgo la relación médico-paciente, la que se puede ver afectada al enfrentar al niño a tomas de muestras de sangre. Otra ventaja de usar saliva como fluido de muestra es que permite detectar la fracción libre de un fármaco (no unida a proteína), lo que es importante, ya que su acción terapéutica depende de esta fracción ${ }^{16}$.

El objetivo del presente estudio fue medir concentraciones de carbamazepina en plasma y en saliva de pacientes epilépticos voluntarios, y relacionar los valores obtenidos para determinar, considerando a éste un estudio piloto, si la medición de niveles del fármaco en saliva podría servir como estimación de sus niveles plasmáticos.

\section{MATERIAL y MÉTODO}

Los sujetos incluidos en este estudio ( $n=11$ ) fueron pacientes que recibían carbamazepina como medicamento para la epilepsia, que se atendían en el Policlínico de Neurología del Hospital Las Higueras de Talcahuano, Chile, que tenían indicado el control de los niveles plasmáticos de este fármaco y que aceptaron participar, previo consentimiento informado de sus padres. Las edades de los pacientes fluctuaron entre $8 \mathrm{y}$ 15 años. Todos llevaban al menos 6 meses de tratamiento con carbamazepina.

Tanto las muestras de saliva como las de sangre fueron tomadas en el Laboratorio Clínico Dr. Raúl Campos, hasta donde llegaron los pacientes voluntarios portando una tarjeta que los identificaba como parte de este estudio y excluyéndolos de la cancelación de los exámenes. Estas tarjetas fueron proporcionadas por el médico tratante. En el laboratorio se les consignaron datos referentes a la edad, dosis del medicamento, fecha y hora de la última dosis antes del examen. Las muestras de saliva fueron obtenidas, sin estimulación, mediante una tórula de algodón introducida en la boca del paciente, la cual posteriormente se colocaba en una jeringa, vaciando el fluido obtenido dentro de un microtubo de centrífuga. Las cantidades así obtenidas fluctuaron en alrededor de 0,5 a $1 \mathrm{~mL}$ de saliva. Las muestras se centrifugaron a 500 rpm por 3 min y se trabajó con el sobrenadante, sin ser necesario algún tratamiento especial.

Las muestras de sangre se obtuvieron en forma simultánea. Se trabajó separando el plasma y colocándolo en tubos con heparina o EDTA. 
Tanto las muestras de sangre como las de saliva se tomaron alrededor de 9 a $11 \mathrm{~h}$ después de la última dosis de carbamazepina.

Las muestras de sangre se analizaron en el laboratorio clínico y las de saliva fueron trasladadas a la Universidad de Concepción en envases aislantes y analizadas por el grupo de investigadores. La determinación de las concentraciones salivales de carbamazepina se realizó mediante un método autográfico por cromatografía planar instrumental (HPTLC) ${ }^{17}$; y la de carbamazepina en plasma mediante un método por inmunoensayo disponible en el Laboratorio Clínico Dr. Raúl Campos.

Los límites de cuantificación fueron de 0,54 ng/uL para el método en saliva por HPTLC, y de $1,01 \mathrm{ng} / \mathrm{uL}$ para el método en plasma por inmunoensayo, por lo que ambos métodos cubren las concentraciones de carbamazepina esperadas en cada fluido biológico (1,2-3,5 ng/uL en saliva y 3$14 \mathrm{ng} / \mathrm{uL}$ en plasma). Esto permite que los resultados obtenidos por una u otra metodología sean comparables. Los valores obtenidos en ambas matrices se relacionaron mediante el coeficiente de correlación obtenido de la curva de las concentraciones del fármaco en ambos fluidos; de la razón de las concentraciones de carbamazepina en saliva y en plasma; y del porcentaje de las concentraciones obtenidas en saliva en relación a las encontradas en plasma.

\section{RESULTADOS}

Las concentraciones plasmáticas de carbamazepina (CBZ) encontradas en plasma estuvieron entre 1,41 y $12,08 \mathrm{ng} / \mathrm{uL}$; y las salivales entre 1,04 y 2,2 $\mathrm{ng} / \mathrm{uL}$, siendo indetectables en el caso del paciente que presentó concentración plasmática de 1,41 ng/uL (Tabla 1). Este valor no se consideró en los estudios de relación entre los niveles de carbamazepina en saliva y plasma, por estar por debajo del límite de cuantificación del método analítico empleado.

El promedio de la razón de las concentraciones de carbamazepina en saliva y plasma fue de 0,18 (intervalo entre 0,11 y 0,29, sin considerar el paciente cuya concentración salival de carbamazepina fue indetectable), con una desviación estándar de 0,05. El promedio de las relaciones porcentuales entre las concentraciones del medicamento en plasma y saliva fue de $17,97 \%$ (desviación estándar $=5,40$ ), por lo cual podría obtenerse una concentración aproximada de carbamazepina en saliva mediante la relación: [CBZ saliva] $=0,18$ [CBZ plasma].

Sin embargo, no se encontró una buena correlación entre las concentraciones de carbamazepina en plasma y saliva ( $r=0,37$ ) (Figura 1). Según estudios de docimasia de hipótesis, considerando el $\mathrm{t}$ de Student como estadístico de prueba y un $\alpha=0,05$, se obtuvo un tc $=1,13$ y un $t$

Tabla 1. C oncentraciones de carbamazepina en saliva y plasma según paciente

\begin{tabular}{|ccc|}
\hline Paciente & $\begin{array}{c}\text { Carbamazepina en saliva } \\
(\mathrm{ng} / \mathrm{uL})\end{array}$ & $\begin{array}{c}\text { Carbamazepina en plasma } \\
(\mathrm{ng} / \mathrm{uL})\end{array}$ \\
\hline 1 & 2,20 & 12,08 \\
2 & 1,65 & 7,06 \\
3 & 1,73 & 7,84 \\
4 & 1,46 & 9,52 \\
5 & 1,35 & 9,52 \\
6 & 1,42 & 4,85 \\
7 & 1,04 & 7,18 \\
8 & 1,54 & 9,52 \\
9 & No detectable & 1,41 \\
10 & 1,45 & 9,52 \\
11 & 1,28 & 11,30 \\
\hline
\end{tabular}




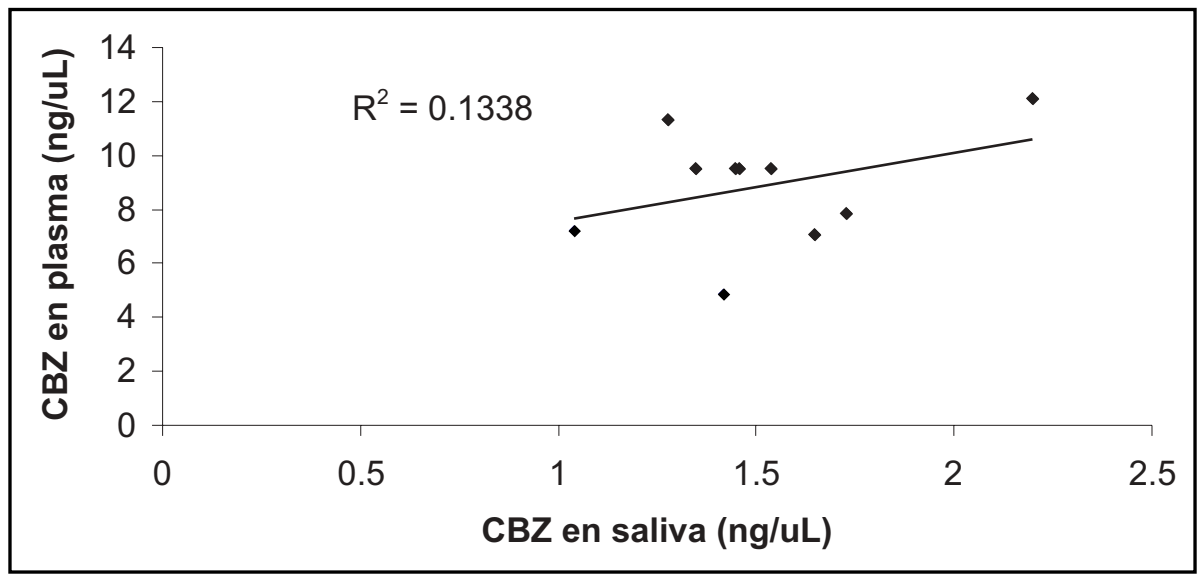

Figura 1. Correlación entre concentraciones de carbamazepina (CBZ) en saliva y plasma.

tab $=1,86$, lo que indica una falta de correlación significativa entre las concentraciones del fármaco en ambas matrices biológicas $(\mathrm{n}=10)$.

\section{DISCUSIÓN}

Para la totalidad de los pacientes incluidos en el estudio, las dosis de carbamazepina estuvieron entre 300 y $800 \mathrm{mg} /$ día, lo que está dentro de los rangos habituales de $400 \mathrm{mg} /$ día a $600 \mathrm{mg} /$ día para niños entre 5 a 10 años, y de $0,6 \mathrm{~g} /$ día a 1,0 $\mathrm{g} /$ día para niños entre 10 a 15 años ${ }^{8}$.

El intervalo de concentraciones plasmáticas de carbamazepina encontradas en los pacientes en este estudio estuvo entre 1,41 y 12,08 ng/uL De este rango, un paciente presentó concentraciones plasmáticas de 1,41 ng/uL, y el resto tuvo concentraciones entre 4,85 y $12,08 \mathrm{ng} / \mathrm{uL}$ El intervalo terapéutico en plasma se ha informado entre $3 \mathrm{y}$ $14 \mathrm{ng} / \mathrm{uL}^{2,8}$, lo que indica que todos los pacientes, salvo uno, presentaba concentraciones plasmáticas útiles del medicamento. En el caso del paciente cuyo nivel de carbamazepina estaba bajo el rango terapéutico, se postula que esto pudo deberse a falta de cumplimiento de la terapia, interacción con otros medicamentos, intervalo de tiempo transcurrido entre la última dosis del medicamento y la toma de muestra, o factores fisiológicos propios del paciente, ya que la dosis del fármaco estuvo dentro de los rangos norma$\operatorname{les}^{8}$ y fue de $600 \mathrm{mg} /$ día. Las concentraciones de carbamazepina encontradas en saliva estuvieron entre 1,04 y 2,2 ng/uL, con la salvedad que fueron indetectables para el paciente que presentó concentración plasmática de 1,41 ng/uL; esto debido a la proporción entre concentraciones del medicamento en plasma y saliva y al límite de cuantificación del método analítico $(0,54 \mathrm{ng} / \mathrm{uL})^{17}$. Este límite de cuantificación indica una muy buena sensibilidad del método, lo que sugiere que las concentraciones de carbamazepina en saliva para ese paciente eran cercanas a cero.

Las concentraciones óptimas de carbamazepina en saliva se han informado entre 1,2 y $3,5 \mathrm{ng} /$ $\mathrm{uL}^{18}$. Esto indica que todos los pacientes, salvo uno, presentaban concentraciones de carbamazepina en saliva dentro del rango terapéutico. El valor de carbamazepina en saliva en el paciente en el cual fue indetectable, así como su concentración en plasma, no fueron consideradas en el estudio de relación entre ambas matrices biológicas.

En relación a la razón de las concentraciones de carbamazepina en saliva y plasma, ésta fue de 0,18 en promedio. La desviación estándar obtenida para el cálculo de las razones de las concentraciones en saliva y plasma fue bastante baja $(0,05)^{16,19}$. Sin embargo, el índice de correlación (r) entre las concentraciones del medicamento en 
plasma y saliva fue de 0,37 , lo que indica una falta de correlación significativa entre los niveles de carbamazepina en ambos fluidos para la muestra estudiada. La utilización de las concentraciones de fármacos en saliva para predecir sus concentraciones plasmáticas es una atractiva alternativa a la determinación directa en plasma por varias razones. La obtención de muestras de saliva es un método sencillo y no invasivo, lo que permite realizar monitoreos terapéuticos y estudios farmacocinéticos en poblaciones especiales como por ejemplo niños y ancianos, sin someterlos a pinchazos repetidos. Otra ventaja es que las concentraciones de los fármacos en saliva reflejan la fracción libre de éste, no unida a proteínas, cuál es su fracción activa terapéuticamente. Esta área representa posibilidades de futuras investigaciones y más estudios clínicos de correlación entre concentraciones de fármacos en plasma y saliva.

Investigaciones anteriores realizadas por diversos autores han mostrado una buena correlación entre niveles plasmáticos y salivales de acetaminofeno o paracetamol, ácido salicílico, teofilina y tolbutamida ${ }^{20}$, y también se han determinado niveles en saliva de quinolonas ${ }^{21-24}$, algunos analgésicos mayores ${ }^{25}$, ciertos antimicóticos ${ }^{26-28}$ y metotrexato ${ }^{29}$, entre otros.

El presente trabajo es un estudio piloto debido al reducido tamaño de la muestra. Sería deseable

\section{REFERENCIAS}

1. GarnetT W. Antiepileptic drug treatment: outcomes and adherence. Pharmacotherapy 2000; 20 (8Pt2): 191S-199S.

2. HaRdman J, Limbird L Goodman \& Gilman. Las bases farmacológicas de la terapéutica, $10^{\mathrm{a}} \mathrm{Ed}$., Mc Graw-Hill, México, 2003.

3. Liu H, Delgado M, Forman LJ, Eggers CM, Montoya JL. Simultaneous determination of carbamazepine, phenytoin, phenobarbital, primidone and their principal metabolites by high-performance liquid chromatography with photodiodearray detection. J Chromatography 1993; 616: 105-15.

4. Delgado J, Remers W. Wilson/Gisvolds. Textbook of organic medicinal and pharmaceutical chemistry, $10^{\mathrm{a}}$ Ed, Lippincott-Raeven, Philadelphia, 1998. aumentar el número de pacientes en el futuro para poder así obtener una conclusión definitiva respecto a la correlación entre los niveles de carbamazepina en saliva y plasma, y concluir si la determinación de la concentración de este medicamento en saliva es un estimador confiable de sus niveles en plasma.

La limitante en la obtención de un mayor tamaño de muestra en el presente estudio, fue la motivación de los pacientes o de las personas a su cargo, ya que, a pesar de haber sido un estudio sin costo para ellos, la mayoría no concurrió al laboratorio clínico para la toma de muestra. Tal vez una forma de obtener un mayor tamaño de muestra sería trabajar con una población cautiva, por ejemplo, pacientes hospitalizados, cuidando de evitar aquellos que tengan politerapia, por las interacciones que pudieran presentarse entre otros medicamentos y carbamazepina.

En cuanto al efecto de la etnia y el género en la farmacocinética de la carbamazepina, éstos no han sido evaluados sistemáticamente, por lo que también resulta de utilidad realizar estudios en nuestra población, ya que la mayoría de los datos existentes se refieren a la población estadounidense o europea.

Los autores esperan que éste y otros estudios al respecto estimulen a realizar investigaciones en el área.

5. Ryan N, Bathara V, Perel J. Mood stabilizers in children and adolescents. J Am Acad Child Adolesc Psychiatry 1999; 38: 529-36.

6. PAGe C. Farmacología Integrada, Harcourt, Madrid, 1999.

7. Drug Evaluations Annual 1995, American Medical Association, 1995.

8. Mc Evoy G. AHFS Drug Information, 47a Ed., American Hospital Formulary Service, USA, 2005.

9. Brody T, Larner J, Minneman K. Human pharmacology molecular to clinical, 3a Ed., Mosby-Year book, Inc., St Louis, USA, 1998.

10. Bhatti M, Hanson G, Schultz L. Simultaneous determination of phenytoin, carbamazepine and 10,11-carbamazepine epoxide in human plasma by high performance liquid chromatography with ultraviolet detection. J Pharm Biomed Anal 1998; 16: 1233-40. 
11. Gholami K, Farahani FB, Zarghi A. Simultaneous determination of carbamazepine and its major metabolite in serum. Pharm Pharmacol Comm 1998; 4: 197-9.

12. Olano I, VásQuez M, Fagiolino P. Chronopharmacokinetics of carbamazepine and its metabolite 10,11-epoxide. J Pharm Clin 1998; 17: 153-6.

13. FEREY K. Simultaneous determination of carbamazepine and epoxy-carbamazepine in plasma by HPLC. J Pharm Clin 1997; 16: 136-40.

14. Fowler J, Ryan M, Kasarkis E. Phenytoin monitoring of patients in a long-term care facility. Hospital Pharmacy 1998; 33: 1097-101.

15. JimÉnEZ M, DuRÁn J, AbAdin J. Factors affecting the free plasma fraction of phenyotin in patients with epilepsy. Clin Drug Invest 1998; 15: 153-7.

16. Pichini S, Altterl I, Zuccaro P, Pacifici R. Drug monitoring in nonconventional biological fluids and matrices. Clin Pharmacokinet 1996; 30: 21128.

17. Mennickent S, Vega M, Godoy CG. Development and validation of a method using instrumental planar chromatography for quantitative analysis of carbamazepine in saliva. J Chil Chem Soc 2003; 48: 71-3.

18. Rylance G, Molreland T. Saliva carbamazepine and phenytoin level monitoring. Arch Dis Child 1981; 56: 637-52.

19. TOMLIN PI, MCKINLAY I, SMITH I. A study on carbamazepine levels, including estimation of 10 11 epoxy-carbamazepine and levels in free plasma and saliva. Develop Med Child Neurol 1986; 28: 713-8.

20. Cook CE, Amerson E, Poole K, Lesser P, O'Tuama L. Phenytoin and phenobarbital concentrations in saliva and plasma measured by radioinmunoassay. Clin Pharmacol \& Ther 1975; 18: 742-5.

21. Stass H, Dalhoff A, Kubitza D, Schuhly V. Pharmacokinetics, safety and tolerability of ascending single doses of moxifloxacin, a new 8-methoxy quinolone, adminitered to healthy subjects. Antimicrob Agents Chemoter 1998; 42: 2060-5.

22. Zhai S, Wei $X$, Parker B, KunZe K, Vestal R. Relation between plasma and saliva concentrations of enoxacin, ciprofloxacin, and teophylline. Ther Drug Monit 1996; 18: 666-71.

23. Ohkubo T, Suno M, Kudo M, Uno T, Sugawara K. Column-switching high-performance liquid chromatography of ofloxacin in human saliva and correlation of ofloxacin level in saliva and serum. Ther Dug Monit 1996; 18: 598-603.

24. Nakashima M, Vematsu T, Kosuge K, Okuyama Y, Morino A, OzaKi M et al. Pharmacokinetics and safety of NM441, a new quinolone, in healthy male volunteers. J Clin Pharmacol 1994; 34: 9307.

25. Ortelu D, Rudaz S, Chevaley A-F, Mino A, Deglon J-J, BaLANT L ET AL. Enantioselective análisis of methadone in saliva by liquid chromatographymass spectrometry. J Chromatography A 2000; 871: 163-72.

26. García-Hermoso D, Dromer F, Improvisi L, Provost F, Dupont B. Fluconazole concentrations in saliva from AIDS patients with oropharyngeal candidosis refractory to treatment with fluconazole. Antimicrob Agents Chemother 1995; 39: 656-60.

27. CodD JE, DESAY PB. Formulation, development and in vivo evaluation of a novel bioadhesive lozenge containing a synergistic combination of antifungal agents. Int J Pharm 1998; 173: 13-24.

28. L S, My Hu FQ. Determination of tinidazole (TNZ) in saliva by HPLC after administration. Chin J Pharm Anal 1997; 17: 307-9.

29. Press J, Berkovitch M, LaXer R, Giesbrecht E, Silverman E, KieIn K ET al. Evaluation of therapeutic drug monitoring of methotrexate in saliva of children with rheumatic diseases. Ther Drug Monit 1995; 17: 247-50.

Agradecimientos

A la Dirección de Investigación de la Universidad de Concepción a través del Proyecto DIUC № 201.074.026-1.0. Al Laboratorio Clínico Dr. Raúl Campos. 